\title{
ÍNDICE PERCIBIDO DE LA CALIDAD AMBIENTAL Y ESTRÉS AMBIENTAL EN ESTUDIANTES DE LA UNMSM
}

\author{
PERCEIVED INDEX OF THE ENVIRONMENTAL QUALITY AND \\ ENVIRONMENTAL STRESS IN STUDENTS OF THE UNMSM
}

\author{
HÉCTOR M. HERNÁNDEZ VALZ ${ }^{1}$, JOSE C. RIVERA BENAVIDES, MILDRED T. \\ PAREDES TARAZONA \\ Universidad Nacional Mayor de San Marcos, Facultad de Psicología
}

\begin{abstract}
RESUMEN
OBJETIVO: Relacionar el índice percibido de la calidad ambiental con el estrés ambiental en estudiantes de la UNMSM - Perú. DISEÑO:

Descriptivo correlacional con una muestra intencional de 200 sujetos. MATERIAL Y MÉTODOS: Se usó el diferencial semántico para medir ambas variables. Se midieron 5 aspectos del ambiente seleccionados por la muestra: la basura, áreas verdes, el smog, el tránsito, la seguridad, siendo el SMOG considerado el más grave. RESULTADOS: Se encontró un índice PEQI igual a 3,82, ligeramente satisfactorio. El nivel de estrés ambiental medido fue de una media de 3,45, poco satisfactorio. CONCLUSIONES: Ambos resultados indican una relación poco buena con el entorno. La relación entre ambas variables tiene una $r$ igual a 0,62 , es decir que es significativa.
\end{abstract}

Palabras clave: PEQI, estrés ambiental, Diferencial semántico.

\begin{abstract}
OBJECTIVE: To relate the perceived index of the environmental quality to environmental stress in students of the UNMSM - Peru. DESIGN: Descriptive corelational with an intentional sample of 200 subjects. MATERIAL And METHODS: The semantic differential was used to measure both variables. 5 aspects of the atmosphere were moderate selected by the sample: the green sweepings, areas, the smog, the transit, the security, being the considered SMOG most serious. RESULTS: Was an equal index PEQI to 3.82, slightly satisfactory. The level of measured environmental stress was of an average of 3.45, little satisfactory. CONCLUSIONS: Both results little indicate a good relation with the surroundings. The relation between both variables has an equal $r$ to 0.62 , is to say that it is significant.
\end{abstract}

Keywords: PEQI, Differential, environmental stress, semantic differential

${ }^{1}$ Email:hhvalz@yahoo.com 


\section{INTRODUCCIÓN}

La necesidad de establecer si los habitantes de un determinado entorno encuentran en aquellos elementos necesarios para que no se produzcan efectos nocivos sobre la salud, tanto a corto como a mediano plazo, asignándole la responsabilidad de interactuar para mantener los diferentes estímulos y sustancias que lo afectan en límites adecuados llevó a desarrollar la medición de la calidad ambiental mediante técnicas de medición de actitudes, como el 0.5. (Hollahan, 1997). ${ }^{1}$

Esta investigación se enmarca en la psicología ambiental, la cual se interesa en conocer el producto de la interacción ser humano - entorno, en términos de calidad de vida expresada en la posibilidad de reducir los factores que contribuyen a deteriorar la salud mental, estén presentes o no daños fisiológicos.

El índice percibido de la calidad ambiental En primer lugar se debe definir la calidad ambiental. Este concepto tiene sus orígenes en la teoría mediacional2 y la técnica derivada de dicha teoría para su aplicación a la medición del significado connotativo, denominada diferencial semántico. Bajo dicha concepción, la calidad ambiental es un significado connotativo perteneciente a un grupo cultural (Díaz Guerrero ${ }^{3}$ ) Se trata de estimar cuan saludable es un entorno a partir de la subjetividad del grupo que vive en condiciones ambientales específicas, y es por tanto una medida netamente psicológica.

El PEQI es por tanto un análogo del diferencial semántica destinado a evaluar estímulos ambientales en condiciones naturales o de simulación. Su construcción requiere de la construcción de los adjetivos bipolares apropiados al habla de la población a ser examinada mediante los métodos señalados por Osgood, Succi y Tannebaun2

Entre los antecedentes tenemos la referencia de Hollahan1, de aplicaciones a la evaluación de paisaje en ambiente de carreteras y arquitectónicos.

Con respecto a el estrés ambiental y la relación entre percepción de la calidad ambiental y salud, el estrés es la percepción de una situación de amenaza frente a la cual se responde con un nivel de ansiedad y fatiga (en calidad de respuesta mediacional. (Osgood, 1972) 2 . Incluye un componente orgánico y otro psicológico. La respuesta ante el estrés es de dos tipos: Respuesta enfocada al problema que consiste en actos cognoscitivos dirigidos hacia la fuente del estrés para modificar la condición ambiental que lo provoca o la conducta personal para enfrentarla, o ambas. La respuesta también puede estar orientada a los estados emocionales, es decir, a racionalizar la amenaza, lo cual produce conflictos subliminales a nivel del sistema nervioso vegetativo, que se acumulan y resultan perjudiciales a mediano plazo. Este último tipo de respuesta es un tema que en años recientes ha generado una campo de estudio denominado psiconeuroinmunología 1,4 pero que hasta la fecha no ha sido vinculado ni directa ni indirectamente con el ambiente, debido a un reduccionismo clínico. En ese sentido nuestro enfoque se centra en la destrucción de barreras interdisciplinarias para favorecer una visión integral del aquí y el ahora de las vivencias cotidianas sin desconocer la historia individual.

De aquí que en futuras investigaciones tendremos que relacionar las condiciones del comportamiento ambiental con la neuropsicoinmunología.

Los objetivos de la investigación son los siguientes:

- Medir el índice percibido de la calidad ambiental en estudiantes de la UNMSM

- Medir el estrés ambiental en estudiantes de la UNMSM

- Establecer la relación entre el índice percibido de calidad ambiental y el estrés ambiental en estudiantes de la UNMSM 
La hipótesis que se desea despejar en la investigación es:

Existe relación entre el índice percibido de la calidad ambiental y el estrés ambiental en estudiantes de la UNMSM.

\section{MATERIALES Y MÉTODOS.}

- Investigación descriptiva correlacional

- Muestra intencional de 200 estudiantes.

- Estadísticas descriptivas y correlación de Pearson.

\section{Instrumentos}

- Diferencial semántica (tabla 1) elaborado a partir del castellano de estudiantes universitarios, elaborado en dos etapas para medir la calidad ambiental.

Se construyó en dos etapas, solicitando a una muestra de estudiantes universitarios que señalen los adjetivos que están más relacionados con los problemas ambientales. Y luego que le asigne los antónimos. Se eligieron los de mayor frecuencia, asignados a cada una de las dimensiones del Diferencial Semántica:

Evaluación, Actividad, Potencia y Familiaridad. Este conjunto de pares se aplicó a cada uno de los factores ambientales previamente elegidos por la misma población constituyendo los siguientes enunciados :

La basura en donde vivo es

Las áreas verdes donde vivo son

El smog donde vivo es

La seguridad donde vivo es

El tránsito donde vivo es

\begin{tabular}{|c|c|l|}
\hline Dimensión & Variable & \multicolumn{1}{|c|}{ Descripción } \\
\hline \multirow{5}{*}{ Evaluación } & F1 & inutil-útil \\
& F2 & intolerable-tolerable \\
& F4 & injusta-justa \\
& F5 & antisocial-social \\
& inaceptable-aceptable \\
\hline \multirow{5}{*}{ Potencia } & F7 & débil-fuerte \\
& F8 & subalterno-dominante \\
& F9 & sébil-poderosa \\
& F10 & no importante-emocionante \\
\hline \multirow{5}{*}{ Actividad } & F11 & estática-dinámica \\
& F12 & pesada-ágil \\
& F13 & lenta-veloz \\
& F14 & retrasadora-aceleradora \\
& F15 & desalentadora-alentadora \\
\hline \multirow{5}{*}{ Familiaridad } & F16 & apartada-vecina \\
& F17 & desconocida-conocida \\
& F18 & separada-adyacente \\
& F19 & infrecuente-común \\
& F20 & mundana-doméstica \\
\hline
\end{tabular}


Se utilizó una escala de seis grados en la que polarizan los grados del extremo.

Este diferencial semántico fue aplicado a un total de 200 estudiantes de la UNMSM de las diferentes

- Escala de cuatro factores para el análisis de las dimensiones afectivas:

Agrado, Activación, Impacto y Control. (tabla2)

Consta de 16 pares de adjetivos que fueron seleccionados entre los correspondientes a cada factor para formar la escala.

Tabla 2: Escala de Estrés Ambiental

\begin{tabular}{|c|c|}
\hline Factor 1: Agrado & Factor 3: Impacto \\
Agradable - Desagradable & Mayor - Menor \\
Repulsivo - Atractivo & Inmenso - Diminuto \\
Incómodo - Confortable & Débil - Fuerte \\
Acogedor - Inhóspito & Grandioso - Insignificante \\
\hline Factor 2: Activación & Factor 4: Control \\
Silencioso - Bullicioso & Complejo - Sencillo \\
Poblado - Desértico & Seguro - Inseguro \\
Muerto - Vivo & Oscuro- Claro \\
Desierto - Dormido & Tranquilo - Intranquilo \\
\hline
\end{tabular}

\section{Análisis de datos}

El análisis de los datos se estructura en torno a tres tipos de puntuaciones, similares a las que se obtienen en la analítica de las escalas del diferencial semántico. Estas puntuaciones son las siguientes:

- Puntuación factor. Es la medida de las puntuaciones obtenidas por cada sujeto en los pares de adjetivos correspondientes a cada factor. Por ejemplo, la puntuación en el factor agrado será igual a la media de las puntuaciones obtenidas en los pares «agradabledesagradable», «repulsivo-atractivo», «incómodo-confortable» y «acogedor-inhóspito». Y así se calculan las cuatro puntuaciones factor (agrado, activación, impacto y control) para cada uno de los sujetos.

- Polarización. Esta puntuación es menos importante, pero puede ser útil en algunos casos. Es la raíz cuadrada de la suma de cada una de las puntuaciones factor elevadas al cuadrado, de esta forma:

$$
\text { Po }=\text { Raíz Cuadrada }\left(\text { Agrado }^{2}+\text { Activación }^{2}+\text { Impacto }^{2}+\text { Control }^{2}\right)
$$

- Distancias semánticas. Esta puntuación pretende registrar las diferencias entre las puntuaciones en los cuatro factores obtenidas en a) o bien dos grupos de sujetos o bien en b) dos conceptos o lugares distintos. La fórmula se basa en el cálculo de las diferencias de medias.

$$
\mathrm{D}_{\mathrm{s}(1-2)}=\text { Raíz Cuadrada }\left(\left(\mathrm{a}_{1}-\mathrm{a}_{2}\right)^{2}+\left(\mathrm{ac}_{1}-\mathrm{ac}_{2}\right)^{2}+\left(\mathrm{i}_{1}-\mathrm{i}_{2}\right)^{2}+\left(\text { có́ }_{1}-\mathrm{có}_{2}\right)^{2}\right.
$$




\section{Procedimientos de aplicación de la escala}

Una vez elegidos los lugares que van a ser analizados, se pueden diseñar dos procedimientos de aplicación de la escala:

a) Procedimiento de evaluación en contexto natural y

b) Procedimiento de evaluación en contexto de simulación.

El procedimiento a) se refiere a la colaboración de usuarios del lugar que mientras que el b) consiste en la evaluación de diapositivas seleccionadas. Para esta investigación era necesario elegir el procedimiento a), por cuanto remite al sujeto en su entorno habitual o lugar de residencia.

Por tal razón el modelo de consigna que recibieron los sujetos evaluados fue la siguiente:

Instrucciones: Se está realizando un estudio sobre el significado del espacio. Por favor, piense un momento en el lugar en el que vive y los lugares por los que comúnmente transita o realiza sus actividades laborales o de estudios y luego caracterizará la impresión que generalmente le causan graduando su respuesta según cada uno de los pares de adjetivos que se le presentan con una $\mathrm{X}$ en alguna de las opciones entre cada par.

\section{RESULTADOS}

- Determinación de los factores ambientales de mayor impacto sobre la población estudiada, mediante encuesta: Basura, Tránsito, Áreas Verdes, Smog y Seguridad.

- Elaboración y validación del diferencial semántico para el PEQI, con estudiantes de diferentes universidades

- Análisis de validez y confiabilidad.

La confiabilidad fue determinada mediante el método de la consistencia interna con la fórmula $\mathrm{Kr}_{20}$ y se hallaron los siguientes resultados

Tabla 3: Coeficientes de Confiabilidad

\begin{tabular}{|c|c|}
\hline & \\
Basura & 0.67 \\
Transito & 0.74 \\
Áreas verdes & 0.82 \\
Smog & 0.64 \\
Seguridad & 0.91 \\
\hline
\end{tabular}


Aplicación del D.S. a la muestra de la UNMSM:

Gráfica 1: Diagnéstico del Indice Percibido de la Calidad

Ambiental y Fistres Ambiental en Estudiantes UNMSM

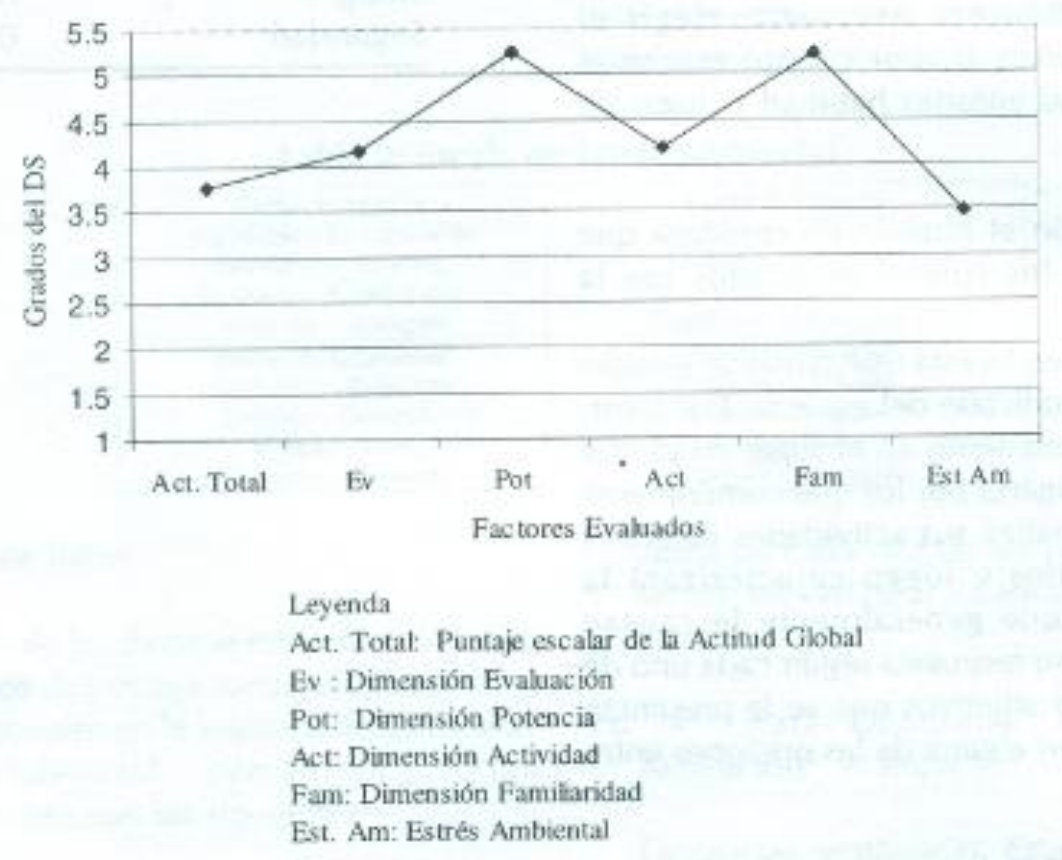

El índice Percibido de la Calidad Ambiental (PEQIS)

De acuerdo con la técnica del diferencial semántica debe señalarse la actitud a partir de cada una de las dimensiones. Al respecto se han efectuado los siguientes hallazgos:

\section{DIMENSIÓN EVALUACIÓN:}

Cae en el grado 4, con tendencia a favorable y que tiene el significado de estar conforme con el entorno siempre y cuando no se oriente hacia la afirmación de que las cosas ya están bien. Es una percepción no totalmente explicita de que algunos aspectos no son los adecuados. Existe un grado de atracción que hace tolerable la situación.

\section{DIMENSION POTENCIA}

Cae en el grado 5, con una fuerte tendencia a aceptar que las cosas están prácticamente dadas y que es poco lo que se puede hacer por lograr una transformación. Corresponde a considerar que hay pocas fuerzas para lograr algún cambio.

\section{DIMENSIÓN ACTIVIDAD}

Cae en el grado 4, con una tendencia favorable en cuanto a provocar en la muestra evaluada ciertos niveles de movilidad, pero es menor que el resultado en la dimensión Potencia. 


\section{DIMENSION FAMILIARIDAD}

Cae en el grado 5, indicando que la muestra evaluada tiene conocimiento y está inmersa dentro del ambiente evaluado. Se puede precisar que no muestra extrañamiento y que está en condiciones de reconocer la problemática o al menos algunas características.

\section{EL INDICE PERCIBIDO DE LA CALIDAD AMBIENTAL (PEQIS)}

Ubicado en el grado 4, es, en general, ligeramente favorable, es decir que el estudiante universitario encuentra un nivel de bienestar más bien favorable a partir del entorno en el que vive.

\section{EL ESTRÉS AMBIENTAL}

Cae en el grado 3, con tendencia a sufrir relativamente poco estrés como consecuencia del entorno, en todo caso el sujeto no es suficientemente consciente de riesgos derivados de la problemática ambiental, o que se encuentra con ellos sólo en determinados momentos.

- Aplicación de la escala de estrés ambiental.

\begin{tabular}{|c|c|c|c|c|c|c|c|c|c|}
\hline \multirow[t]{2}{*}{$\begin{array}{l}\text { Media } \\
\text { jenenal }\end{array}$} & & & & \multirow[b]{2}{*}{3} & \multirow[b]{2}{*}{4} & \multirow[b]{2}{*}{5} & \multirow[b]{2}{*}{6} & & \multirow[t]{2}{*}{$\begin{array}{l}\text { Media por } \\
\text { Area }\end{array}$} \\
\hline & & 1 & 2 & & & & & & \\
\hline 3,45 & Desagradahle & & & 9 & & & & Agradable & \multirow{7}{*}{3,43} \\
\hline 3,37 & Repelsivo & & & & & & & Atractivo & \\
\hline 3,39 & Incómodo & & & 6 & & & & Confortahle & \\
\hline 3,49 & Inhóspito & & & & & & & Acogedor & \\
\hline 3.75 & Bulliciose & & & 9 & & & & Silencioso & \\
\hline 3,78 & Desértico & & & 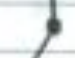 & & & & Poblado & \\
\hline 3,53 & Muento & & & & & & & Vivo & \\
\hline 3,47 & Dormido & & & $\oint$ & & & & Despierto & \multirow[t]{4}{*}{3,63} \\
\hline 3,41 & Menor & & & F & & & & Mayor & \\
\hline 3.14 & Diminuto & & & & & & & Inmenso & \\
\hline 3,47 & Oscuro & & & 9 & & & & Claro & \\
\hline 3,37 & Insignificanie & & & $\oint$ & & & & Grandiosso & \multirow[t]{4}{*}{3,35} \\
\hline 3,41 & Complejo & & & 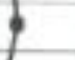 & & & & Sencillo & \\
\hline 3,33 & Inseguro & & & 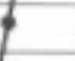 & & & & Seguro & \\
\hline 3,24 & Debia & & & & & & & Foente & \\
\hline \multirow[t]{3}{*}{3,55} & Intranquilo & & & 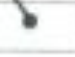 & & & & Tranquilo & \multirow[t]{3}{*}{3,38} \\
\hline & Media & 3,45 & & & & & & & \\
\hline & Desv Est. & 0,16 & & & & & & & \\
\hline
\end{tabular}

Los resultados presentados en la tabla 2 ilustran que hay un grado moderado de estrés y que en ningún par de adjetivos se produce polarización. Lo cual tampoco se da a nivel de cada una de las cuatro áreas. En este sentido las respuestas tienden a ser conservadoras y con una apariencia de «más o menos", antes que reconocer francamente que hay un problema o de expresar la ausencia de problema. 
- Correlación entre El D.5. Para el PEQI la escala de estrés ambiental.

Tabla 4: Coeficiente de correlación entre el total de la Actitud (PEQIS), las dimensiones de la estructura EPA y el Estrés Ambiental.

\begin{tabular}{|c|c|c|c|c|c|c|}
\hline & TOTAL PEQIS & Evaluación & Potencia & Actividad & Familiaridad & Estrés \\
\hline TOTAL PEQIS & 1 & & & & & \\
\hline Evaluación & 0,66 & 1 & & & & \\
\hline Potencia & 0,66 & 0,30 & 1 & & & \\
\hline Actividad & 0,70 & 0,29 & 0,20 & 1 & & \\
\hline Familiaridad & 0,67 & 0,14 & 0,36 & 0,33 & 1 & \\
\hline Estrés & 0,09 & 0,06 & 0,10 & 0,04 & 0,04 & \\
\hline
\end{tabular}

Como se puede observar, la actitud total PEQIS, correlaciona significativamente con sus componentes, que conforman la estructura EPA (evaluación, potencia y actividad más familiaridad) estableciéndose así la validez del instrumento. Por otra parte, dichos componentes también presentas correlaciones moderadas pero significativas, estableciéndose que son partes del mismo conjunto.

Con respecto a la escala de estrés ambiental, se puede apreciar la ausencia de relación lo cual indica una independencia entre los factores que estresan a la muestra evaluada y la calidad que atribuyen a la urbe en que viven.

\section{CONCLUSIONES}

- El PEQI encontrado en los estudiantes de la UNMSM Tiene los siguientes valores medios

- Áreas verdes $\quad 4,44$

- El tránsito $\quad 4,11$

- La seguridad $\quad 3,80$

- La basura $\quad 3,47$

- El smog $\quad 3,30$

- Es decir que en el 2001 el problema más agudo era el de el smog o contaminación del aire.

- El PEQI estimado es igual a 3,82, ligeramente favorable porque no llega a ser polarizador.

- El estrés ambiental encontrado tiene una media de 3,45, lo cual implica un estado de parcial insatisfacción ante estímulos no bien identificados.

- La relación entre el PEQI y el estrés ambiental es significativa al 0,05. 


\section{ANEXO \\ ESCALA DE ESTRÉS AMBIENTAL \\ INSTRUCCIONES:}

Se está realizando un estudio sobre el significado del espacio. Por favor, piense un momento en el lugar en el que vive y los lugares por los que comúnmente transita o realiza sus actividades laborales o de estudios y luego caracterizará la impresión que generalmente le causan graduando su respuesta según cada uno de los pares de adjetivos que se le presentan con una $\mathrm{X}$ en alguna de las opciones entre cada par.

\begin{tabular}{|c|c|c|c|c|c|c|c|}
\hline & $\begin{array}{l}\text { Muy } \\
\text { poco }\end{array}$ & Bastante & $\begin{array}{l}\text { Poco } \\
\text { menos }\end{array}$ & $\begin{array}{l}\text { Poco } \\
\text { más }\end{array}$ & Bastante & Mucho & \\
\hline Agradable & & & & & & & Desagradable \\
\hline Silencioso & & & & & & & Bullicioso \\
\hline Mayor & & & & & & & Menor \\
\hline Complejo & & & & & & & Sencillo \\
\hline Repulsivo & & & & & & & Atractivo \\
\hline Poblado & & & & & & & Desértico \\
\hline Inmenso & & & & & & & Diminuto \\
\hline Seguro & & & & & & & Inseguro \\
\hline Incómodo & & & & & & & Confortable \\
\hline Muerto & & & & & & & Vivo \\
\hline Débil & & & & & & & Fuerte \\
\hline Oscuro & & & & & & & Claro \\
\hline Acogedor & & & & & & & Inhóspito \\
\hline Despierto & & & & & & & Dormido \\
\hline Grandioso & & & & & & & Insignificante \\
\hline Tranquilo & & & & & & & Intranquilo \\
\hline
\end{tabular}

hhvalz@yahoo.com

hectorhernandez@mail2world.com

hhernandezv@unmsm.edu.pe

\section{REFERENCIAS}

1. Hollahan, Psicología ambiental: Un enfoque general, México, 1995, Edil. Trillas

2. Osgood Ch, Succi, Tannebaum, La medida del significado, Madrid, 1968, Edil. Gredas

3. Diaz Guerrero R, El Diferencial semántica del idioma español, México, 1975, Edil. Trillas

4. Rivera J. C, Competencias cognitivo afectivas en la educación ambiental, en Revista de Investigaciones Psicológicas, Lima, 2002, UNMSM 